\title{
Bridging the Gap between Nanomagnetic Devices and Circuits
}

\author{
Michael Niemier ${ }^{1}$, X. Sharon $\mathrm{Hu}^{1}$, Aaron Dingler ${ }^{1}$, M. Tanvir Alam ${ }^{2}$, G. Bernstein ${ }^{2}$, and W. Porod ${ }^{2}$ \\ (1) Department of Computer Science and Engineering, (2) Department of Electrical Engineering \\ University of Notre Dame \\ Notre Dame, IN 46556, USA \\ Email: \{mniemier, shu, adingler, malam1, gary.h.bernstein.1, porod $\} @$ nd.edu
}

\begin{abstract}
This paper looks at designing circuit elements that will be constructed with nanoscale magnets within the Quantum-dot Cellular Automata (QCA) computational paradigm. In magnetic QCA (MQCA) logical operations and dataflow are accomplished by manipulating the polarizations of nanoscale magnets. Wires and gates have already been experimentally demonstrated at room temperature. However, to realize more complex circuits - and eventually systems more than just wires and gates in isolation are required. For example, gates must be inter-connected, signals must cross, etc. All structures must be controlled by the envisioned drive circuitry. In this paper, structures that will facilitate these circuit-level tasks are presented for the first time.
\end{abstract}

\section{INTRODUCTION}

At present, numerous research efforts are seeking a new logic device either to replace or augment CMOS technology to continue the performance scaling trends of the past 40 years. This paper looks at how one implementation of the Quantum-dot Cellular Automata (QCA) device architecture might help in this regard. We extend existing experimental work - where wires and gates have been demonstrated in isolation - and consider the structures required to interconnect wires and gates to form more complex circuits. We also consider how said structures will interact with the onchip drive circuitry currently envisioned for systems based on magnetic QCA (MQCA).

QCA accomplishes logical operations and moves data via nearest-neighbor interaction rather than with electric current flow [1]. In MQCA, wires, gates, and inverters have all been realized, they operate at room temperature [2], and [3] estimates that if $10^{10}$ magnets switch $10^{8}$ times/second, they would only dissipate about $0.1 \mathrm{~W}$ of power. When the drive circuitry is included, [4] predicts that circuits could provide performance wins over state-of-the-art, low power CMOS when considering energy delay product. Devices can scale and remain non-volatile provided their size/shape remains above the superparamagnetic limit. However, binary state in nanomagnets with feature sizes below the superparamagnetic limit can be stable for around $1 \mathrm{~ms}$ [5] - long enough to perform logical operations. Scaling can also decrease switching times [5].

Fabricating MQCA-based systems should be fairly straightforward. Nanomagnets can be made using conventional lithography or by leveraging electron beam lithography and liftoff to form specific patterns of magnetic material. One could also leverage imprint lithography where imprint is used to make molds, and then the molds can be used to make the nanomagnet shapes. At least a subset of these methods are compatible with, and can take advantage of, advances in CMOS fabrication techniques.

While efforts to date have experimentally demonstrated the components required for a functionally complete logic set, there is still a large gap between basic MQCA devices and computationally interesting MQCA systems. Our goal is to narrow this gap by designing circuit structures that are essential for building MQCA systems. Here, we use physicallevel simulation to demonstrate how external stimuli (i.e the clock) will affect the logical state of individual magnets of various sizes and shapes. We will leverage this study to (a) ensure that all of the structures required to build a circuit can be controlled with implementable, on-chip drive circuitry, (b) design structures that allow data to flow orthogonally to the direction of the applied clock, and (c) design structures for crossing two logical signals with nanomagnets.

\section{BACKGROUND}

\section{A. QCA Circuit Constructs}

The initial description of a QCA device called for encoding binary numbers into cells that have a bi-stable charge configuration [1]. A QCA device would consist of 2 or 4 "charge containers" (i.e. quantum dots) and 1 or 2 excess charges respectively. One configuration of charge could represent a binary '1' and the other a binary '0' - with logical operations and data movement accomplished via Coulomb (or nearest-neighbor) interactions. In MQCA, charge configurations are replaced with magnetic polarizations of single domain magnets.

Schematic representations of an MQCA wire and gate appear in Fig. 1a-b. A wire is just a line of magnets that are antiferromagnetically coupled to one another. The basic logic gate is based on the majority voting function - where the output of the gate is the logical value associated with the majority of the 3 input cells. Note that by setting one input of a majority gate to a logic ' 0 ' or ' 1 ', the gate will execute an AND or OR function respectively. In MQCA, the gate performs an inverting majority voting function. Both of these structures have been experimentally demonstrated at room temperature (see Fig. 1c,d [2]).

\section{B. Required Clock Functionality}

The structures illustrated in Figs. 1c,d were tested with a "clock" that took the form of a periodically oscillating, external magnetic field that drove a system to an initial state, and then controlled the relaxation of the said system to a 


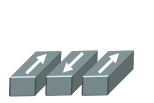

(a)

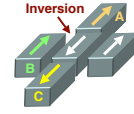

(b)

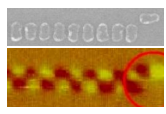

(c)

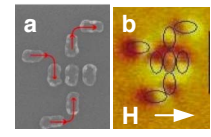

(d)
Fig. 1. Cartoon representations of (a) a wire segment and (b) a majority gate. Wire segments (c) and majority gates (d) have been experimentally demonstrated.

(a)
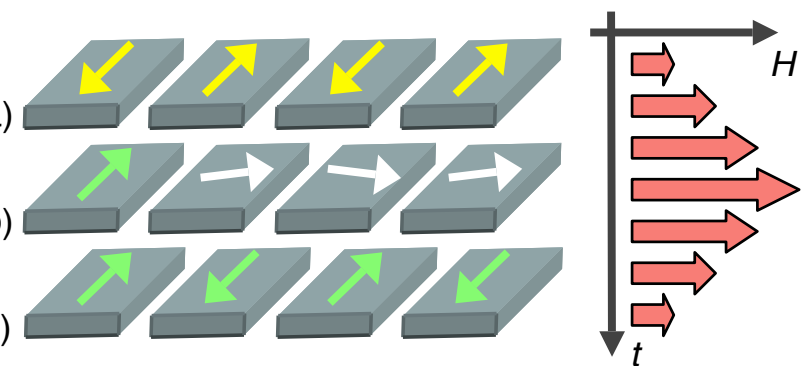

Fig. 2. How we might change the logical state of a wire: (a) initial, logically correct, antiferromagnetically ordered ground state; (b) a new input is applied to the line and an external magnetic field is applied to the system which polarizes magnets along their hard axes; (c) the second magnet in the line, influenced by the new input, switches to a new, logically correct state, which switches the third magnet, etc. and the applied field is removed.

ground state. More specifically, a magnetic field is applied along the hard (or shorter) axes of a group of nanomagnets. As an example, Fig. 2a illustrates a line of nanomagnets that are initially in a logically correct, antiferromagnetically coupled ground state. In Fig. 2b, the external field turns the magnetic moments of each magnet horizontally into a neutral logic state against the preferred magnetic anisotropy. In this system, this is an unstable state. When the field is removed, the nanomagnets should relax into an antiferromagnetically ordered state but in accordance with the new polarization of the first magnet in the line (Fig. 2c). Thus, the first magnet in this line provides the biasing field that eventually sets the state of the entire line via bipolar coupling.

\section{Clock Implementation}

[4] explored the use of copper wires wrapped in ferromagnetic material on the sides and bottom to provide onchip, local control for MQCA circuits. According to the behavior of magnetic circuits, a magnetic field, $\mathrm{H}$, wraps around the current-carrying wire, inducing a constant flux, $\phi$, whose density, B, depends on the space through which it passes. In traversing a path around the clock wire, the flux threads both the magnetic materials (i.e. the ferrite and nanomagnets) and the intervening gaps (i.e. the oxide or air). Maximizing the fraction of the total distance that the flux travels in the high-permeability materials increases the field intensity, and therefore the flux density, in the remaining gaps. With this arrangement, local fields of approximately $4,000 \mathrm{~A} / \mathrm{m}$ to $40,000 \mathrm{~A} / \mathrm{m}$ could be produced in $2 \mu \mathrm{m}$ wide, $4 \mu \mathrm{m}$ long, and $200 \mathrm{~nm}$ thick ferrite-yoked wires with current densities of $10^{6} \mathrm{~A} / \mathrm{cm}^{2}$ and $10^{7} \mathrm{~A} / \mathrm{cm}^{2}$ respectively [4].

\section{Analyzing Magnetic Circuit Elements}

Here, we present a methodology for analyzing magnetic circuit elements that can be used to help generate initial designs and to evaluate circuit-level functionality and robustness. We leverage the OOMMF simulation suite [6] created by NIST, which integrates the Landau-Lifshitz equation. OOMMF is widely used and there is excellent correlation between simulation and experimental results (see [7], [8]).

\section{A. Magnetization State - Traditional MH Curves}

For an elongated, high aspect ratio nanomagnet, in the absence of an applied magnetic field, a magnet's magnetization, M, will tend to align along its easy (i.e. long) axis $(\uparrow$ or $\downarrow$ ) due to shape anisotropy. This bistable state can be used to encode a single bit of information where a positive $M_{y}$ represents a binary 0 and a negative $M_{y}$ a binary 1 .

If we want to switch the magnetization state, we must apply a strong bias in the y-direction opposite to its initial magnetization. The minimum field along the $\mathrm{y}$-axis required to perform this switching is $H_{\text {bias }}=\left(N_{x}-N_{y}\right) M_{s}$, where $N_{x}$ and $N_{y}$ are the demagnetizing factors along the hard and easy axes respectively (see Sec. IV for more detail), and $M_{s}$ is the saturation magnetization [9].

As an example, an external field of approximately 130,000 $\mathrm{A} / \mathrm{m}$ would need to be applied along the easy axis of a $60 \times 90 \times 20 \mathrm{~nm}^{3}$ permalloy magnet in order to change its magnetization state. That said, in an MQCA-based circuit, the biasing field required to switch the device needs to come from a neighboring input magnet. However, an equivalently sized device placed $20 \mathrm{~nm}$ away from the magnet that we want to switch would realistically only generate an $H_{\text {bias }}$ of approximately $10,000 \mathrm{~A} / \mathrm{m}$ when we average the $\mathrm{y}$ component of magnetization in the next magnet's "footprint." The disparity between the biasing field required and the biasing field a magnet can produce can be addressed by introducing an applied field $\left(H_{x}\right)$ along a magnet's hard axis (i.e. its short axis) that helps to facilitate the switching process (see Sec. II-B). From Fig. 3, it is clear that as $H_{x}$ increases, the magnitude of the biasing field required to switch this magnet is reduced considerably.

\section{B. Magnetization State - MQCA Representation}

Throughout this paper, we will present the data captured by Fig. 3 via a non-traditional hysteresis curve. Namely, we will consider the y-component of magnetization $\left(M_{y}\right)$ as a function of the applied field $\left(H_{x}\right)$ - as this analysis inherently includes information about logical correctness/binary state $\left(M_{y}\right)$ as well as the local clocking field $\left(H_{x}\right.$ - hereafter referred to as $H_{\text {clock }}$ ). Before discussing specific designs, we use this analysis to show how we can leverage magnetic material to more easily change the polarization of neighboring nanomagnets. This facilitates tasks such as dataflow in a line of nanomagnets that is orthogonal to the direction of the clocking field.

More specifically, we present the results of two simulations (see Fig. 4) performed with the OOMMF simulation suite. All simulations consider a $60 \times 90 \times 30 \mathrm{~nm}^{3}$ supermalloy magnet (the material used in [10]) initially oriented down 


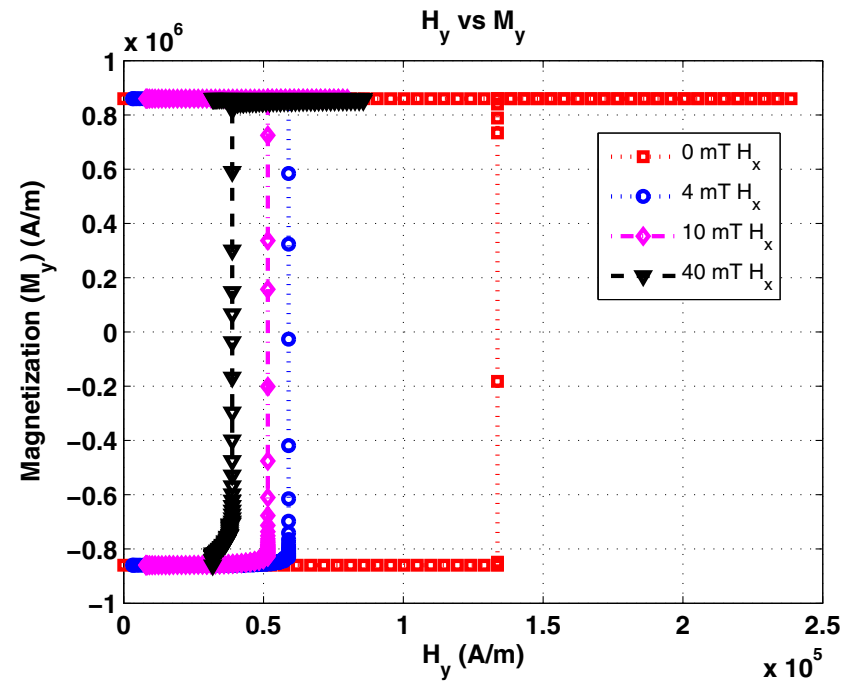

Fig. 3. For a $60 \times 90 \times 20 \mathrm{~nm}^{3}$ permalloy magnet in isolation, note that as the magnitude of the external field $\left(H_{x}\right)$ increases, the magnitude of the biasing field (applied in the y-direction) required to flip the magnet decreases.

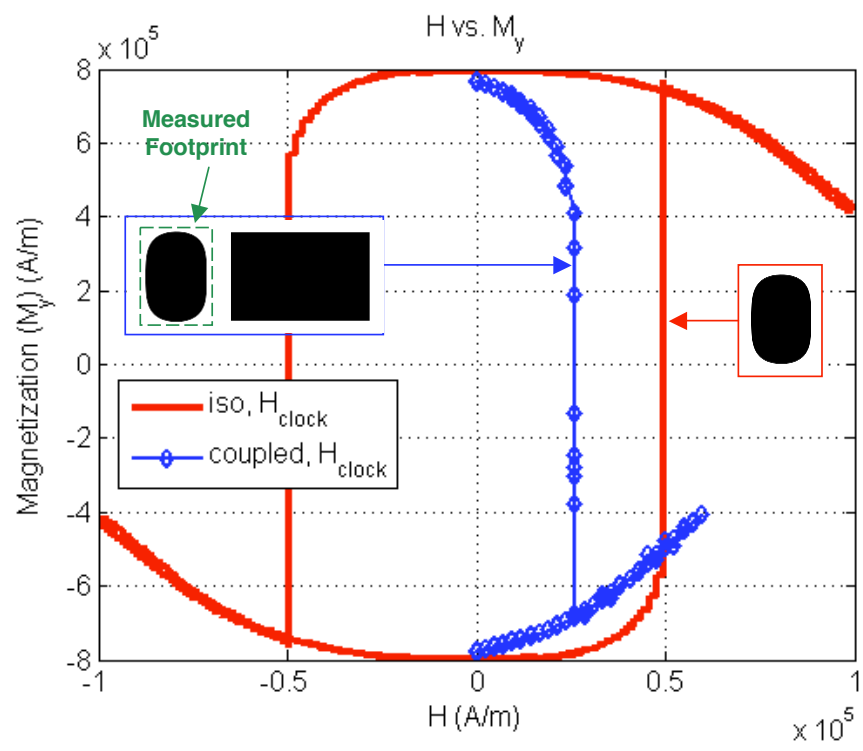

Fig. 4. The simulation described by a solid line curve without markers illustrates the external field required to null a $60 \times 90 \times 30 \mathrm{~nm}^{3}$ magnet in isolation. The line with markers considers the same magnet coupled with a block of magnetic material. A lower external field is required to facilitate a state change.

$(\downarrow)^{1} . H_{\text {clock }}$ is applied in the positive x-direction (i.e., leftto-right along the magnet's hard axis). A constant 15,000 $\mathrm{A} / \mathrm{m}$ biasing field is applied along the magnet's easy axis which "tips" the magnet to the opposite polarization.

We begin by considering a single magnet in isolation (see "iso, $H_{\text {clock" }}$ in Fig. 4). The magnet is initially oriented down and its y-component of magnetization is strongly negative (at saturation $M_{y}=-800,000 \mathrm{~A} / \mathrm{m}$ ). An external field $\left(H_{\text {clock }}\right)$ is then applied along the hard axis of this magnet.

\footnotetext{
${ }^{1} \mathrm{As}$ in [11], we assumed a saturation magnetization of $8.0 \times 10^{5} \mathrm{~A} / \mathrm{m}$, an exchange stiffness constant of $1.05 \times 10^{-11} \mathrm{~J} / \mathrm{m}$, an anisotropy constant $K_{1}$ of $3 \mathrm{~J} / \mathrm{m}^{3}$, and the default damping constant of 0.5 . Each simulation stage (when the magnitude or direction of the applied field changes) was considered complete when the maximum $|d \mathbf{m} / d t|$ dropped below a preset number of degrees per nanosecond.
}

As $H_{\text {clock }}$ increases, the magnitude of the y-component of magnetization decreases - eventually reaching 0 when $H_{\text {clock }}$ is approximately $50,000 \mathrm{~A} / \mathrm{m}$.

Note that in the "iso, $H_{\text {clock}}$ " curve in Fig. 4, we continue to increase the magnitude of $H_{\text {clock }}$ even after $M_{y}$ changes signs (indicating a $\downarrow$ to $\uparrow$ transition). The net result is that $M_{y}$ is suppressed and actually begins to decrease (see the "tail" at the top-right of the "iso, $H_{\text {clock}}$ " curve). Only when $H_{\text {clock }}$ is eventually relaxed does $M_{y}$ again saturate (time at positive $800,000 \mathrm{~A} / \mathrm{m}$ ). In the second half of this simulation, we repeat the process discussed above, but this time change the sign of $H_{\text {clock }}$ to simulate an external field applied in the opposite direction. Note that the second half of "iso, $H_{\text {clock" }}$ in Fig. 4 is essentially symmetrical to the first half.

These results suggest two very important things. First, the applied field can be too strong. In the simulation discussed above, the biasing field is externally generated, but in an MQCA-based circuit it would come from the nanomagnets in the circuit. While an external nulling field can assist with switching and the evaluation of new inputs, it can also impede dataflow by suppressing the ability of a magnet to switch its neighbor (as the biasing field generated by the magnet will be of lower magnitude). Second, the symmetry of the "iso, $H_{\text {clock" }}$ " curve suggests that the direction of $H_{\text {clock }}$ should not affect the direction of dataflow. The state that a magnet switches to is determined solely by the biasing field of a neighboring magnet. Thus, a unidirectional clock is sufficient to facilitate bi-directional dataflow. Feedback should be possible within a single clock wire group.

While the main focus of this work is not to optimize the energy efficiency of a given design, we cannot neglect the fact that the "iso, $H_{\text {clock" }}$ " simulation seemingly indicates that an external field of at least approximately 50,000 A/m is require to null/switch a magnet (irrespective of the biasing field). If a field of this magnitude were truly necessary, [4] suggests that a current density of over $10^{7} \mathrm{~A} / \mathrm{cm}^{2}$ would be required. This would not only dramatically increase the overall system energy, but heating and electromigration effects would be a practical concern.

That said, the magnets and magnetic material that comprise a circuit can actually assist in the switching process which will allow us to decrease the magnitude of $H_{\text {clock }}$. To illustrate, we again consider a $60 \times 90 \times 30 \mathrm{~nm}^{3}$ magnet, but this time add a block of magnetic material $25 \mathrm{~nm}$ to the right of it. The block of magnetic material has an aspect ratio that is less than 1 and its easy axis is parallel to the applied field. The first half of the simulation discussed earlier in this section (for "iso, $H_{\text {clock" }}$ ") was repeated with the same $15,000 \mathrm{~A} / \mathrm{m}$ bias. Looking at the "coupled, $H_{\text {clock" }}$ " curve, one can see that magnet switches when $H_{\text {clock }}$ is approximately $26,000 \mathrm{~A} / \mathrm{m}$ - a field half that required to null/switch a magnet in isolation.

When the block is placed in close vicinity to the nanomagnet, the block concentrates flux towards itself. In other words, as the block is already magnetized along the positive $\mathrm{x}$-direction (due to its shape anisotropy) it increases the magnetic flux density in the $\mathrm{x}$-direction in the surrounding area. This increase in flux density assists the switching 
process as it creates a strong local magnetic field in the $\mathrm{x}$-direction. In fact, we can consider the block used in the 'coupled, $H_{\text {clock }}$ ' simulation in isolation and calculate the average field generated by the block where the magnet eventually resides. Simulation data indicates that the block provides a local field where $H_{x}$ averages to be approximately $37,000 \mathrm{~A} / \mathrm{m}$. Thus, introduction of the block helps reduce the minimum applied field requirement, which ultimately reduces the external energy needed to perform the switching.

We refer to the magnetic field produced by the block magnet and $H_{\text {clock }}$ as $H_{\text {local }}$ (thus $H_{\text {local }}=H_{\text {clock }}+$ $\left.H_{\text {block}}\right)$. The magnitude of $H_{\text {local }}$ clearly will depend on the block size, aspect ratio, shape, material and distance from the nanomagnet. $H_{\text {local }}$ is an important parameter for MQCA circuit design and should be carefully tuned using all available parameters. Although one might be tempted to think that we would like to maximize $H_{\text {local }}$ so that $H_{\text {clock }}$ can be minimized, as seen earlier, if the net field applied to a magnet is too strong, it can keep a magnet magnetized in the $\mathrm{x}$-direction and impede the biasing field that will ultimately switch a neighboring device.

\section{Shape, Size, And Circuits}

While many of the circuit designs discussed here consider sizes and shapes consistent with the work in [4], in the remainder of this paper we will investigate how magnets with other shapes could facilitate the realization of more complex circuit structures. Using the techniques discussed in Sec. III, we provide the necessary background for the work to be discussed in Secs. V, VI, and VII. Size and shape will have an effect on (a) the y-component of magnetization - which Imre, et. al. leverage to represent a binary 1 or 0 , (b) the magnitude and direction of the field produced by a piece of magnetic material - which affects how good of a "driver" a given magnet might be, and (c) how hard or easy it is to null a magnet and/or change its polarization - which will have an effect on the overall energy required to perform computation.

We begin by considering OOMMF simulations of 4 magnets in isolation. Each magnet has a different aspect ratio. For each simulation, we wanted to determine the magnitude of $H_{\text {clock }}$ required to change the magnetization state of each device. 60x90, 60x105, 60x120, and $65 \times 95 \mathrm{~nm}^{2}$ magnets (all $30 \mathrm{~nm}$ thick) were considered with the same $H_{\text {bias }}$ applied to each. As seen in Fig. 5, as a magnet's aspect ratio increases, the magnitude of $H_{\text {clock }}$ required to switch the magnet increases as well (only two curves are included to improve graph readability).

This trend can be explained by the concept of the demagnetizing field. When some material is magnetized in a given direction, the field/flux lines emerge from the North pole and return at the South pole. Some of these field lines pass through the material as well. These field lines are called the demagnetizing field as they have direction opposite to that of magnetization $\mathrm{M}$, and thus they work as a demagnetizing force. The demagnetizing field in a direction is proportional to the magnetization in that direction, $H_{\text {demag }}=N_{d} M$, where the demagnetizing factor, $N_{d}$, depends on the size and shape of the material. In general, $N_{d}$ is lowest along the

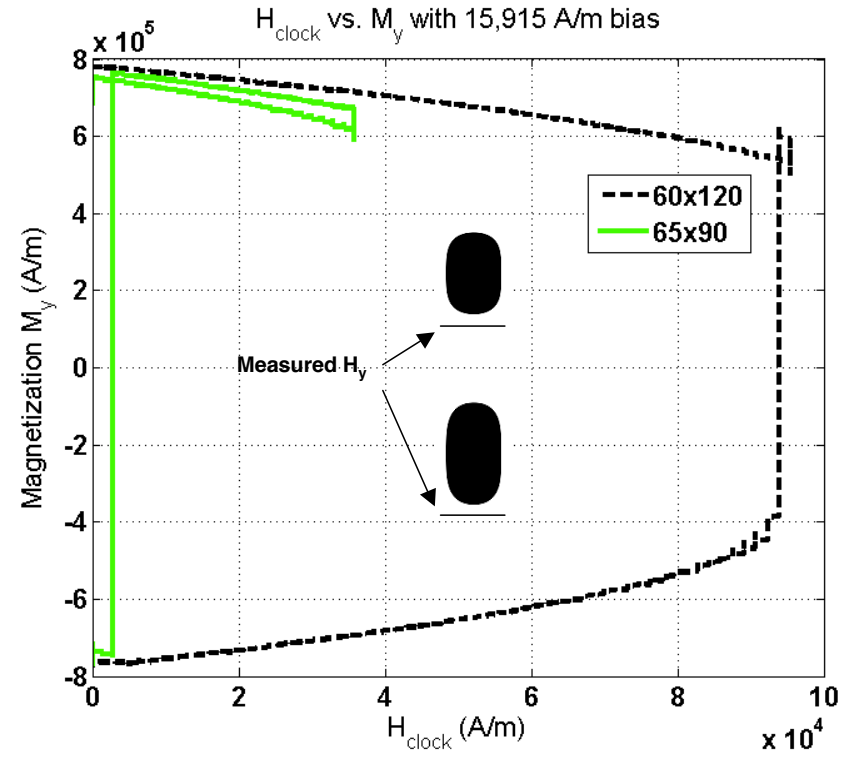

Fig. 5. As a magnet's aspect ratio increases, given a similar biasing field, the magnitude of $H_{\text {clock }}$ required to null/switch the magnet increases.

TABLE I

$M_{y-a v g}$ AND $H_{y-\text { Bias }}$ FOR MAGNETS OF DIFFERENT ASPECT RATIOS.

\begin{tabular}{|c|c|c|c|}
\hline Magnet & $60 \times 90$ & $60 x 105$ & $60 \times 120$ \\
\hline$M_{y-\text { avg }}(\mathrm{A} / \mathrm{m})$ & $-7.71 \times 10^{5}$ & $-7.901 \times 10^{5}$ & $-7.97 \times 10^{5}$ \\
\hline$H_{y-\text { Bias }}(\mathrm{A} / \mathrm{m})$ & $-2.33 \times 10^{5}$ & $-2.48 \times 10^{5}$ & $-2.56 \times 10^{5}$ \\
\hline
\end{tabular}

longest dimension and highest along the shortest dimension [9]. The minimum field needed to align the magnetization of a nanomagnet along its hard axis is given by $H_{\text {clock }}=\left(N_{x}-\right.$ $\left.N_{y}\right) M_{s}$, where $N_{x}$ and $N_{y}$ are the demagnetizing factors along hard and easy axes of the nanomagnet respectively, and $M_{s}$ is the saturation magnetization. As the aspect ratio of a nanomagnet increases, the difference between $N_{x}$ and $N_{y}$ increases and thus the minimum $H_{c l o c k}$ needed to null the magnet increases.

In future work, we will discuss in more detail how to leverage demagnetizing factors to size magnetic material such that we can change its magnetization state given a specific $H_{\text {clock }}$ and/or $H_{\text {bias }}$ of a given magnitude, have a block of magnetic material generate a biasing field of a given magnitude at a specific location, etc. However, for now, we simply leverage simulation data to show how aspect ratio affects how strong of a "driver" a magnet is. To quantify this, we measured the magnitude of the magnetic field produced by each magnet by averaging the field associated with each simulation mesh point $4 \mathrm{~nm}$ directly below a magnet's lower edge (see insets in Fig. 5 for an example). We pay particular attention to the y-component of magnetization $\left(H_{y-b i a s}\right)$ because (as we will see next), it is a good measure of how strong of a "driver" a magnet is. Sample data points are presented in Table I. As a magnet's aspect ratio (and $M_{y}$ ) increases, the y-component of the generated field increases as well. While easier to null, magnets with lower aspect ratios exhibit weaker $1 \mathrm{~s}$ and $0 \mathrm{~s}$ and are also weaker drivers ${ }^{2}$.

For magnets with a small aspect ratio, the magnetization

\footnotetext{
${ }^{2}$ This can also be seen in Fig. 5. As the magnet's aspect ratio increases, its y-component of magnetization increases too.
} 
Field lines

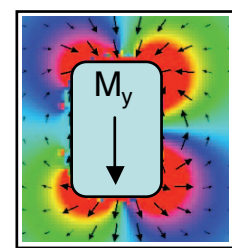

Measured surfaces

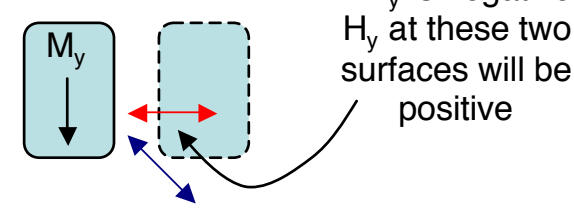

Fig. 6. The magnitude of the y-component of the field produced by a $60 \times 90 \mathrm{~nm}^{2}$ magnet is measured at two different surfaces.

is not totally aligned to its easy, y-axis. As a result, it is not able to produce a strong $H_{\text {bias }}$ along the y-direction, and consequently has a reduced polarization effect on its neighbor magnet. Also, small differences in the demagnetizing factor between the easy axis and the hard axis make the magnetization prone to variations with small field differences along the hard axis.

The above discussion is important as the magnitude of $H_{\text {bias }}$ will have an impact on the required magnitude of $H_{\text {clock}}$. In all of the simulation results discussed thus far, we have essentially biased our magnets by using an artificially generated external field. But as mentioned, $H_{\text {bias }}$ really comes from the magnets themselves. As $H_{\text {bias }}$ increases, the external field required to null the magnet decreases. (One can see this trend graphically by re-examining Fig. 3). From a circuit design perspective, this is important as it suggests that we can reduce the overall system energy by using lower energy drive circuitry if we can generate a stronger local biasing field with magnetic material.

Obviously, a magnet's shape influences the magnitude and direction of the biasing field that it produces. However, how a driver and a magnet being driven are oriented with respect to one another also has an effect. To quantify the magnitude of $H_{\text {bias }}$, we considered the y-component of magnetization of the field generated by an unclocked and unbiased 60x90 nm ${ }^{2}$ magnet in isolation at two different surfaces (see Fig. 6). The surfaces chosen are indicative of where we would want to "see" a given $H_{\text {bias }}$ - perhaps to facilitate antiferromagnetic ordering. Averaging each set of data points, we see that at the horizontal surface, the average y-component of $H_{\text {bias }}$ is $51,600 \mathrm{~A} / \mathrm{m}$ and at the diagonal surface the average $\mathrm{y}$ component of $H_{\text {bias }}$ is $40,200 \mathrm{~A} / \mathrm{m}$. These results suggest that a magnet can generate a rather strong biasing field.

In reality, the biasing field generated by a magnet will be less because it is suppressed by $H_{\text {clock}}$. We can quantify this effect by again applying an external field to the $60 \times 90 \mathrm{~nm}^{2}$ magnet in isolation. Here, no biasing field was applied and we calculated the average magnitude of $H_{b i a s}$ as a function of $H_{\text {clock }}$ for the same surfaces in Fig. 6. As the magnitude of $H_{\text {clock }}$ increases, the magnitude of the biasing field generated by the magnet decreases. For example, when $H_{\text {clock }}=20,000$ $\mathrm{A} / \mathrm{m}$, the average biasing field at the horizontal surface is reduced by nearly $25 \%$ to about $40,000 \mathrm{~A} / \mathrm{m}$. The average biasing field at the diagonal surface is reduced by about $60 \%$ to $25,000 \mathrm{~A} / \mathrm{m}$. Thus, one must consider the magnitude of $H_{\text {clock }}$ when designing circuits if a specific $H_{\text {bias }}$ is required. (Another alternative is to change the geometry of
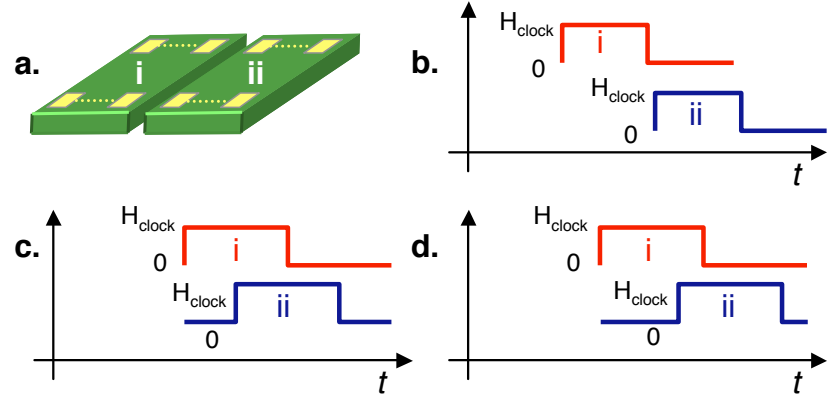

Fig. 7. (a) Different groups of magnets will be controlled by different metal wires as discussed in [4]; (b)-(d) Wires can be active at different times to facilitate dataflow in the nanomagnets.

the nanomagnet to help concentrate flux - increasing the magnitude of $H_{\text {bias }}$ ).

Thus, an ideal device will essentially be easily nulled (with a low magnitude $H_{\text {clock}}$ ), but can also switch state quickly to produce a strong, local $H_{\text {bias }}$ (even if $H_{\text {clock }}$ is still applied). We leverage these ideas to accomplish circuit-level tasks such as facilitating dataflow in a line of magnets across clock wire boundaries and to help move data orthogonally to the direction of $H_{\text {clock }}$.

\section{Clock Wire Boundaries}

All of the elements in an MQCA circuit must be controlled locally, on chip. Before looking at corner turns and crossings, we first consider the interaction between nanomagnets and the drive circuitry in more detail. Of particular interest is how information is transferred in the nanomagnets at the clock wire boundaries (see Fig. 7a). We use a line of nanomagnets as a case study.

The simplest way one could envision controlling a line of nanomagnets that spans multiple clock wires is as proposed in [4] and illustrated in Fig. 7b. Essentially, we would run current through wire (i) to generate a field that would null the first group of nanomagnets, and the magnets in this group would switch in accordance with their input. Next, we would run current through wire (ii) to generate a field that would null the second group of nanomagnets, the first magnet in group (ii) would be biased/driven by the last magnet in group (i), and the rest of the magnets in group (ii) would switch accordingly. Wires (i) and (ii) would not be active simultaneously. We tested this clocking scheme with a short, 12 magnet wire segment (see insets in Fig. 8). The line was initialized to a logically correct, antiferromagnetically coupled ground state and the polarization of the first magnet was flipped to simulate a new input. This input magnet was unclocked. We then applied a 20,000 A/m clocking field to the next 5 magnets and a 20,000 A/m field to the next 6 magnets in succession as illustrated in Fig. 7b. Simulation results are illustrated graphically in inset (a) in Fig. 8. Note that the fourth magnet in the line is frustrated and the last 8 magnets never change state (i.e. a "stuck at" fault ensues).

Why this occurs can be explained by considering the magnetization of the 5th magnet in the line (the first magnet that does not switch) as a function of the magnetization of the 6th magnet in this line (the last magnet in the wire 


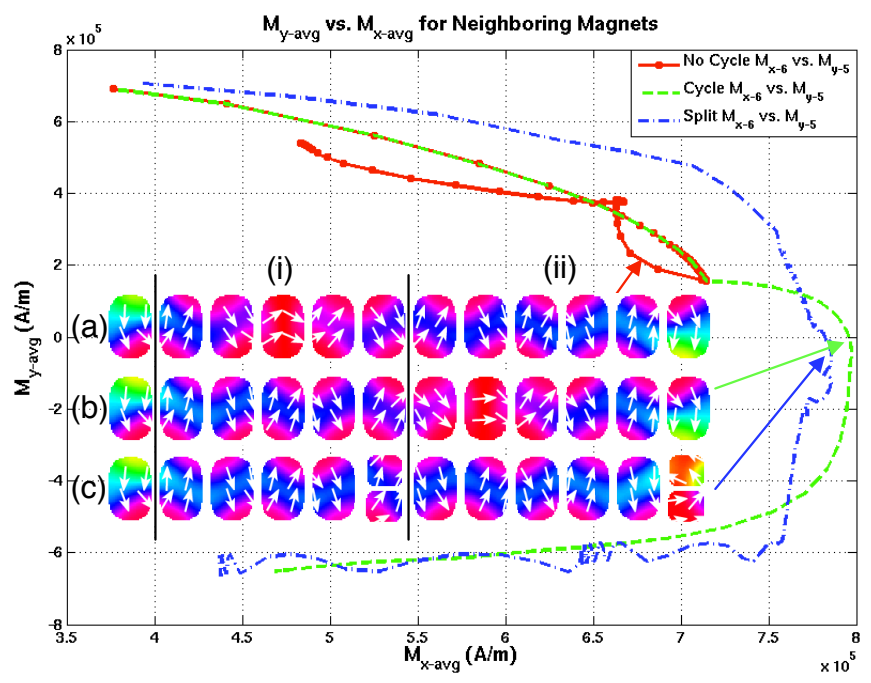

Fig. 8. $M_{y}$ of the 5 th magnet in the line of nanomagnets shown in the insets as a function of $M_{x}$ of the 6th magnet in the line. Note that the magnets in wire group (i) only switch successfully if the magnetic material at the clock wire boundary is sufficiently nulled.

(i) group). More specifically, we consider the y-component of magnetization of the 5th magnet and the x-component of magnetization of the 6th magnet. Examining the "No Cycle $M_{x-6}$ vs. $M_{y-5}$ " curve in Fig. 8, the 6th magnet is never completely nulled (the saturation magnetization for supermalloy is $8.0 \times 10^{5} \mathrm{~A} / \mathrm{m}$ [11]). While a more detailed discussion is beyond the scope of this paper, the unclocked 7 th magnet remains in its initial $\uparrow$ state and essentially provides a sufficiently strong anti-bias which impedes switching of the 6th magnet. This in turn makes it more difficult to null the 5th magnet. While the 5th magnet starts to switch, it is never completely nulled and when $H_{\text {clock }}$ returns to 0 , it relaxes back to its initial state.

Given this result, the clocking methodology proposed in [4] will not allow data to flow in the nanomagnets between groups (i) and (ii) if the wires are excited in the manner summarized in Fig. 7b. However, we have leveraged the simulation results presented in Fig. 4 to develop a modified clock scheme that does allow data to flow across the wire (i)/wire (ii) boundary. Looking back to Sec. III, when we considered a nanomagnet coupled to a block of magnetic material (with the magnetization of the block parallel to the direction of $H_{\text {clock }}$ ), the magnitude of $H_{\text {clock }}$ required for switching to occur was cut in half. While we cannot insert blocks of magnetic material at the clock wire boundaries, we can mimic the effects of the block by exciting both wire (i) and wire (ii) simultaneously as described by Fig. 7c. The result of this simulation is illustrated graphically in inset (b) of Fig. 8. Note that the input to the wire propagates successfully between clock wire groups. Although the signal does become frustrated again in group (ii), this is because we do not control the boundary condition of wire (ii). If a wire group (iii) were to be placed adjacent to wire group (ii), this problem would be eliminated. This simulation is also summarized in Fig. 8. Note that when the 6th magnet is nulled, $M_{y}$ in the 5th magnet switches from positive to negative ('Cycle $M_{x-6}$ vs. $M_{y-5}$ ). By exciting both wire groups simultaneously, (a) the anti-bias produced by the first magnet in wire group (ii) is suppressed and (b) the first magnet in group (ii) contributes to the "nulling" field that the last magnet in the first line experiences.

The downside to this clocking scheme is that it could double the energy associated with the clock - which is already anticipated to be the dominant source of energy in an MQCA-based system. That said, one way to mitigate excess energy consumption is to consider the time evolution of this line. One could determine when the last magnet in a wire group is sufficiently nulled and use this criterion to determine when to stop current flow through wire (i). This might minimize the time that both wires are active simultaneously and lead to the clocking scheme in Fig. $7 d$.

Another alternative is to leverage magnetic material at the clock wire boundaries to facilitate dataflow between clock wire groups. Conceptually, we need a magnet that is both easy to null and an adequate driver. Looking back to Fig. 5, we reported that as a magnet's aspect ratio approached 1 , it became easier to remove the y-component of magnetization and null the magnet. While this comes at the expense of a weaker biasing field (see Table I), such a shape may also be more readily manipulated by the biasing fields of the surrounding magnetic material.

To facilitate dataflow between clock wire boundaries, we leverage magnets with aspect ratios that are less than but still close to 1 . Specifically, we integrate two $50 \times 40 \mathrm{~nm}^{2}$ magnets into the 12 magnet line as shown in inset (c) of Fig. 8. Note that the footprint of this arrangement is approximately that of a $60 \times 90^{2} \mathrm{~nm}$ magnet. However, the easy axis of each $50 \times 40$ $\mathrm{nm}^{2}$ magnet is parallel to the direction of $H_{\text {clock }}$. Hence these magnets should be more easily nulled than a $60 \times 90$ $\mathrm{nm}^{2}$ magnet where shape anisotropy makes it more difficult to magnetize along its hard axis. Next, we examine if the biasing field from a $60 \times 90 \mathrm{~nm}^{2}$ magnet is enough to change the magnetization of the $50 \times 40 \mathrm{~nm}^{2}$ magnets in a direction against that dictated by shape anisotropy.

To determine the magnitude of the biasing field required to orient the $50 \times 40 \mathrm{~nm}^{2}$ magnet up or down, we can perform simulations similar to those in Sec. IV. Namely, we consider a $50 \times 40 \mathrm{~nm}^{2}$ magnet in isolation, allow it to relax, and then apply an external field along its hard axis. As seen in Fig. 9, when the magnitude of the external field is approximately $35,000 \mathrm{~A} / \mathrm{m}$, the $50 \times 40 \mathrm{~nm}^{2}$ magnet has a strong y-component of magnetization ('1 Block' curve). The magnet remains in this state as long as the external field is applied. Otherwise, the magnet would relax back to the state illustrated in the inset of Fig. 9 when $H_{\text {clock }}=0$.

We repeated this simulation, but this time considered two adjacent $50 \times 40 \mathrm{~nm}^{2}$ magnets. The two magnets were initialized such that $M_{x}=800,000 \mathrm{~A} / \mathrm{m}$ and were allowed to relax to a ground state with no applied field (see inset in Fig. 9 - shape anisotropy results in an x-component of magnetization while field lines emanating from each magnet lead to the coupling shown in the inset). We again applied an external field to this arrangement of nanomagnets along their hard axes. These simulation results are also illustrated in Fig. 9. Note that when $H_{\text {clock }}$ is approximately 15,000 


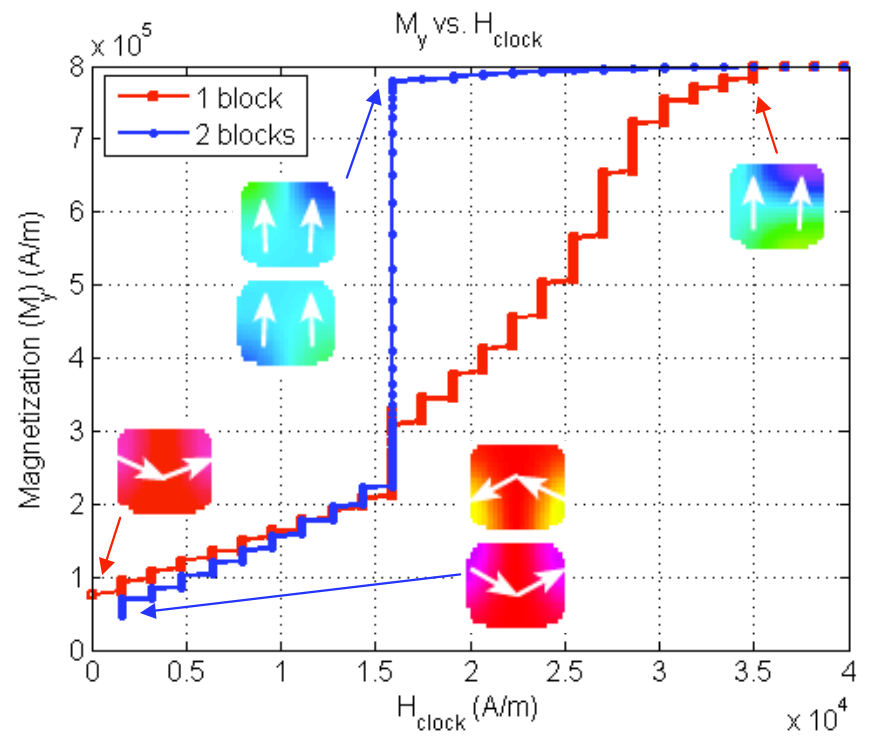

Fig. 9. $M_{y}$ of two different arrangements of nanomagnets as a function of $H_{\text {clock }}$ applied along the magnets' hard axes.

$\mathrm{A} / \mathrm{m}$, this arrangement of nanomagnets has a strong average y-component of magnetization. Looking back to Sec. IV, we noted that even in the presence of an external clocking field, the biasing field produced by a $60 \times 90 \mathrm{~nm}^{2}$ magnet has a magnitude similar to the $15,000 \mathrm{~A} / \mathrm{m}$ field reported here. Thus, this arrangement of $50 \times 40 \mathrm{~nm}^{2}$ magnets could be controlled by the biasing field of another magnet in the line, but also be easily nulled.

We tested this idea by repeating the 12 cell wire simulation with the $50 \times 40 \mathrm{~nm}^{2}$ magnet stack at the clock wire boundaries. The clocking scheme in Fig. $7 \mathrm{~b}$ was used. Simulation results are illustrated by inset (c) in Fig. 8. Here, magnets in both wire groups change in accordance with the new input to produce a logically correct, antiferromagnetically ordered line. Moreover, only one wire group is excited at any given time which will help minimize overall system energy. The $50 \times 40 \mathrm{~nm}^{2}$ magnet stack remains in the state shown in inset (c) in Fig. 8 even when no external field is applied as the $60 \times 90 \mathrm{~nm}^{2}$ magnets adjacent to the stack generate the biasing field needed to preserve antiferromagnetic ordering.

\section{HORIZONTAL AND VERTICAL DATAFLOW}

To form more computationally interesting circuits, gates and wires need to be interconnected. This necessitates more complex dataflow. In this section, we consider the problem of having a horizontal line of nanomagnets drive a signal to a vertical line of nanomagnets with the output of that vertical line driving another horizontal line (i.e. "turning corners"). If we assume that the direction of $H_{\text {clock }}$ is parallel to any horizontal line then we will need to facilitate dataflow in the magnets orthogonal to the direction of the applied $H_{\text {clock }}$.

An obvious attempt to accomplish this task is to use an arrangement of nanomagnets like that shown in Fig. 10a. To test this structure, we initialized the magnets to a logically correct ground state and applied a 20,000 A/m external field. The result is illustrated in Fig. 10b, and the y-component of magnetization of the the last magnet in the vertical line

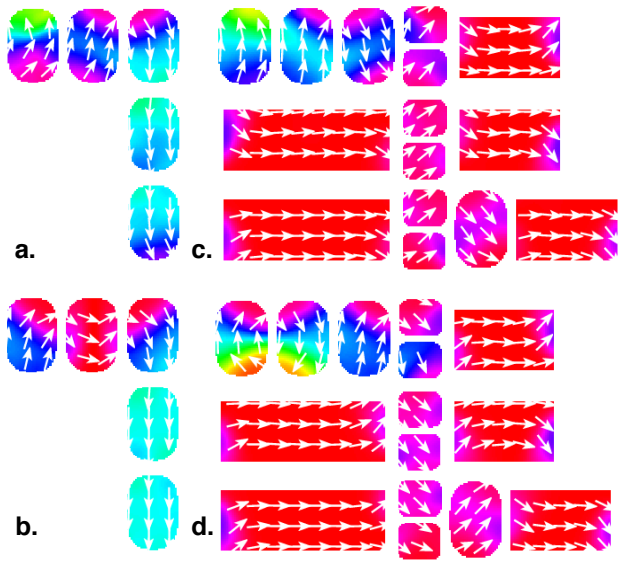

Fig. 10. Vertical dataflow Design 1: before (a), after (b); Vertical dataflow Design 2: before (c), after (d).

appears as a function of time in Fig. 11 (see "No Blocks" curve). Clearly, this structure does not work as intended. For the design in Fig. 10b, the output magnet should be oriented up, but there is little if any change to the y-component of its magnetization. These results can be explained by the simulation results discussed in Fig. 4. The last magnet in this line behaves similarly to that described by "iso, $H_{\text {clock }}$ " in Fig. 4. As it is not coupled to any other magnetic material, the magnitude of $H_{\text {clock }}$ needed to facilitate switching would need to be much larger than $20,000 \mathrm{~A} / \mathrm{m}$. Moreover, given the configuration of magnets in Fig. 10a, the second magnet in the vertical line essentially serves as an anti-bias making it even harder to switch the last magnet.

In an attempt to design a working corner turn, we leveraged the arrangement of $50 \times 40 \mathrm{~nm}^{2}$ magnets discussed at the end of Sec. V and added blocks of magnetic material oriented in the same direction as the applied $H_{\text {clock }}$ to help switch the magnet. Our new design is illustrated in Fig. 10c. Note that the last magnet in the top horizontal line was initially oriented down, the magnets in the vertical wire were all oriented up, and the magnet adjacent to the vertical line in the bottom horizontal line was initially down. Thus, antiferromagnetic ordering was preserved in this initial state. To test this structure, we flipped $M_{y}$ of the first magnet of the top horizontal line and applied a 20,000 A/m external field to this group of magnets. The simulation result appears in Fig. 10d. The magnets in this structure have all switched in accordance with the new input.

While this structure appears to function as intended, ideally, the magnet being driven in the lower horizontal line will be biased such that it represents its new, logically correct state before $H_{\text {clock }}=0$. Otherwise, it might suggest that all dataflow orthogonal to the direction of the clock would need to take place at clock wire boundaries - a significant design constraint. We can determine when the last magnet in the bottom horizontal line switches by considering $M_{y}$ as a function of time (see "Split Magnet" in Fig. 11). Note that $H_{\text {clock }}$ switches from $20,000 \mathrm{~A} / \mathrm{m}$ to $0 \mathrm{~A} / \mathrm{m}$ at $2.61 \times 10^{-9} s$. The last magnet in the lower wire is already biased into its logically correct state and this structure works as intended. 


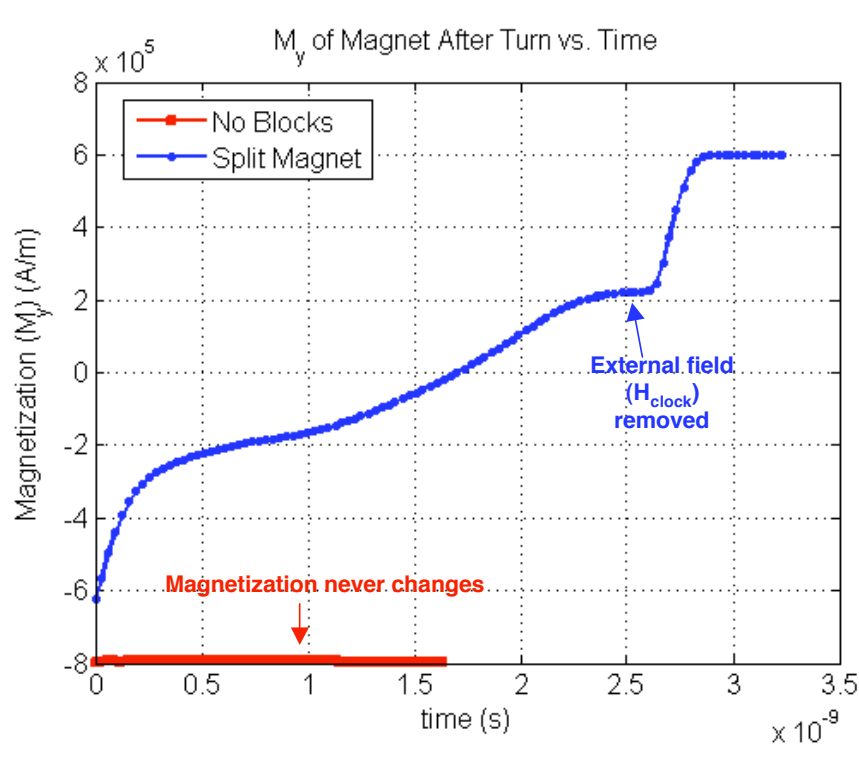

Fig. 11. $M_{y}$ of the output of two corner turn designs as a function of time. Note that for the designs illustrated in Fig. 10, the output is biased into a logically correct state before $H_{\text {clock }}$ relaxes to 0 .

\section{WIRE CROSSINGS}

Here, we show how we can arrange a group of nanomagnets that might function as a wire crossing. We can again leverage the shape of a magnet to represent two bits of information simultaneously. A schematic of what a wire crossing might look like appears in the insets of Fig. 12 - where the middle nanomagnet has a component of both inputs (at left) and transfers the polarization of both inputs to both outputs (at right).

We have leveraged all of the above ideas to design the structure that appears in the Fig. 12 insets (with before and after snapshots). Note that here, the input magnets, output magnets, and middle magnet have all been sized differently. While ultimately this may not be required, sizing helps to ensure that the inputs will have more influence over the device that represents both states, which will in turn have more influence over the output magnets. This helps ensure the correct time evolution of this circuit and prevents "stuck at" states. This is shown quantitatively in Fig. 12 - where we consider both the magnitude and the direction of the local field between the input at bottom-left ('Bottom') and the output at top-right ('Top'). Note that the magnitude of the field between the bottom input and the middle cell is greater than the magnitude of the field between the middle cell and the top output. In future work, we will revisit this design and consider crossovers in conjunction with horizontal and vertical dataflow in the context of more complex circuits.

\section{CONCLUSIONS}

In order to assess the possibility for an emerging technology to replace or augment the existing CMOS technology, it is critical to study circuit and system features in addition to devices. Here, we have examined the construction of several fundamental circuit structures facilitating pipelined signal propagation, multi-direction data flow and co-plane
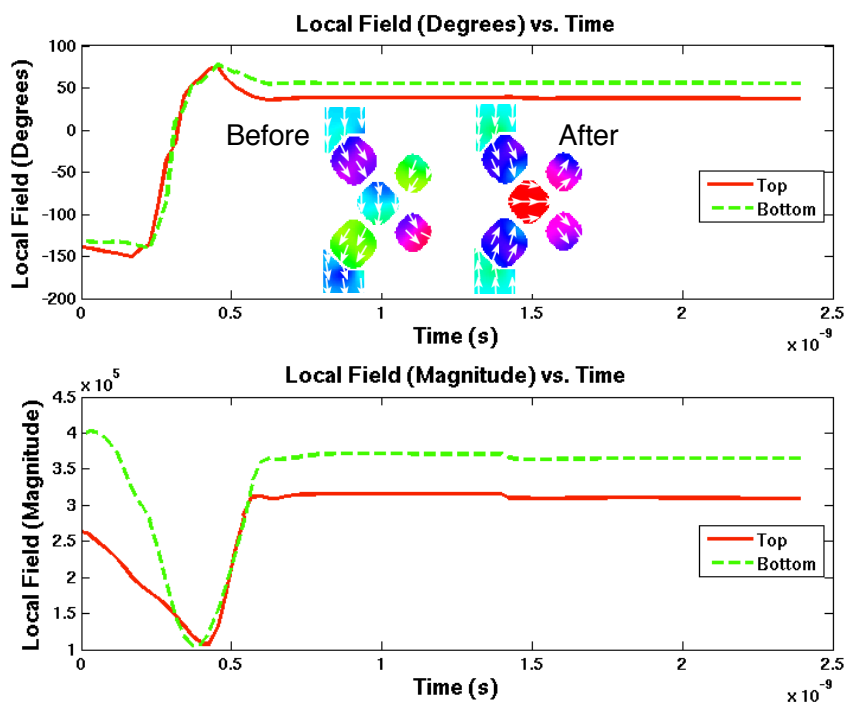

Fig. 12. Magnitude and direction of field between magnets of crossings vs. time.

signal crossing for MQCA. We have demonstrated several plausible designs to accomplish these functions and validated the designs via detailed physical-level simulation. These studies have revealed a number of physical and external design parameters, such as nanomagnet shapes and sizes and clocking field strengths and shapes, which are key to circuitlevel behavior. Careful selection of these parameters helps not only to facilitate computationally interesting circuits, but also can improve circuit performance. Both the methodology and design parameters discussed here will be extremely valuable for further development of MQCA-based systems.

\section{REFERENCES}

[1] I. Amlani, A. Orlov, G. Snider, and C. Lent, "Demonstration of a functional. quantum-dot cellular automata cell," J. Vac. Sci. Tech. B., vol. 16, pp. 3795-99, 1998.

[2] A. Imre, G. Csaba, L. Ji, A. Orlov, G. Bernstein, and W. Porod, "Majority logic gate for Magnetic Quantum-Dot Cellular Automata," Science, vol. 311, No. 5758, pp. 205-208, January 13, 2006.

[3] G. Csaba, P. Lugli, A. Csurgay, and W. Porod, "Simulation of power gain and dissipation in field-coupled nanomagnet," J. of Comp. Electronics, vol. 4(1/2), pp. 105-110, 2005.

[4] M. Niemier, M. Alam, X. Hu, G. Bernstein, W. Porod, M. Putney, and J. DeAngelis, "Clocking structures and power analysis for nanomagnet-based logic devices," Proc. of Int. Symp. on Low Power Elec. and Des. (ISLPED), pp. 26-31, 2007.

[5] X. Wu, C. Liu, L.Li, P. Jones, R. Chantrell, and D. Weller, "Nonmagnetic shell in surfactant-coated FePt nanoparticles," J. Appl. Phys., vol. 95, pp. 6810-6812, 2004.

[6] M. Donahue and D. Porter, "OOMMF User's Guide, Version 1.0, Interagency Report NISTIR 6367,” http://math.nist.gov/oommf.

[7] L. Verma and V. Ng, "Magnetic domain patterns in a zigzag nanowire," J. of Magnetism and Magnetic Materials, vol. 313(2), pp. 317-321, 2007.

[8] S. McVitie, G. White, J.Scott, P. Warin, and J. Chapman, "Quantitative imaging of magnetic domain walls in thin $1 \mathrm{~ms}$ using lorentz and magnetic force microscopies," J. of Appl. Phys., vol. 90(10), pp. 52207, 2001.

[9] R. C. O'Handley, Modern Magnetic Materials: Principles and Applications. New York, NY: John Wiley and Sons, Inc., 2000.

[10] R. Cowburn and M. Welland, "Room temperature Magnetic Quantum Cellular Automata," Science, vol. 287(5457), pp. 1466-1468, February, 2000.

[11] N. Dao, S. Whittenburg, and R. Cowburn, "Micromagnetics simulation of deep-submicron supermalloy disks," J.of Appl. Phys., vol. 90(10), pp. 5235-7, November 15, 2001. 